\title{
On subgraphs induced by transversals in vertex-partitions of graphs
}

\author{
Maria Axenovich \\ Department of Mathematics \\ Iowa State University, Ames, IA 50011, USA \\ axenovic@math.iastate.edu
}

Submitted: Mar 18, 2005; Accepted: Mar 30, 2006; Published: Apr 4, 2006

MR Subject Classifications: 05C15

Keywords: vertex-colorings, Ramsey, induced, transversals, rainbow, multicolored

\begin{abstract}
For a fixed graph $H$ on $k$ vertices, we investigate the graphs, $G$, such that for any partition of the vertices of $G$ into $k$ color classes, there is a transversal of that partition inducing $H$. For every integer $k \geq 1$, we find a family $\mathcal{F}$ of at most six graphs on $k$ vertices such that the following holds. If $H \notin \mathcal{F}$, then for any graph $G$ on at least $4 k-1$ vertices, there is a $k$-coloring of vertices of $G$ avoiding totally multicolored induced subgraphs isomorphic to $H$. Thus, we provide a vertex-induced anti-Ramsey result, extending the induced-vertex-Ramsey theorems by Deuber, Rödl et al.
\end{abstract}

\section{Introduction}

Let $G=(V, E)$ be a graph. Let $c: V(G) \rightarrow[k]$ be a vertex-coloring of $G$. We say that $G$ is monochromatic under $c$ if all vertices have the same color and we say that $G$ is rainbow or totally multicolored if all vertices of $G$ have distinct colors. Investigating the existence of monochromatic or rainbow subgraphs isomorphic to $H$ in vertex-colored graphs, the following questions naturally arise:

Question M: Can one find a small graph $G$ such that in any vertex-coloring of $G$ with fixed number of colors, there is an induced monochromatic subgraph isomorphic to $H$ ? Question M-R: Can one find a small graph $G$ so that any vertex coloring of $G$ contains an induced subgraph isomorphic to $H$ which is either monochromatic or rainbow?

Question R: Can one find a large graph $G$ such that any vertex-coloring of $G$ in a fixed number of colors has a rainbow induced subgraph isomorphic to $H$ ? 
The first two questions are well-studied, e.g., [7], [8], [2]. Together with specific bounds given by Brown and Rödl [3], the following is known:

Theorem 1 (Vertex-Induced Graph Ramsey Theorem). For any graph H, any integer $t, t \geq 2$, there exists a graph $R_{t}(H)$ such that if the vertices of $R_{t}(H)$ are colored with $t$ colors then there is an induced subgraph of $R_{t}(H)$ isomorphic to $H$ which is monochromatic. Let the smallest order of such a graph be $r_{t}(H)$. There are constants $C_{1}$, $C_{2}$ such that

$$
\left.C_{1} k^{2} \leq \max \left\{r_{t}(H)\right):|V(H)|=k\right\} \leq C_{2} k^{2} \log _{2} k \text {. }
$$

The topic of the second question belongs to the area of "canonization", see, for example, a survey by Deuber [5]. The following result of Eaton and Rödl [6] provides specific bounds for vertex-colorings of graphs.

Theorem 2 (Vertex-Induced-Canonical Graph Ramsey Theorem). For any graph $H$, there is a graph $R_{\text {can }}(H)$ such that if $R_{\text {can }}(H)$ is vertex-colored then there is an induced subgraph of $R_{\text {can }}(H)$ isomorphic to $H$ which is either monochromatic or rainbow. Let the smallest order of such a graph be $r_{\text {can }}(H)$. There is a constant $C$ such that

$$
C k^{3} \leq \max \left\{r_{\text {can }}(H):|V(H)|=k\right\} \leq k^{4} \log k
$$

In this paper we initiate the study of Question $\mathrm{R}$ when the number of colors in the coloring corresponds to the number of vertices in a graph $H$. We call a vertex-coloring using exactly $k$ colors a $k$-coloring. In this manuscript we consider only simple graphs with no loops or multiple edges.

Definition 3. For a fixed graph $H$ on $k$ vertices, let $f(H)$ be the maximum order of a graph $G$ such that any coloring of $V(G)$ in $k$ colors has an induced rainbow subgraph isomorphic to $H$. Note that $f(H) \geq k$.

Since a vertex-coloring of $G$ gives a partition of vertices, finding a rainbow induced copy of a graph $H$ corresponds to finding a copy of $H$ induced by a transversal of this partition. Note that $f(H)=\infty$ if and only if for any $n_{0} \in \mathbb{N}$ there is $n>n_{0}$ and a graph $G$ on $n$ vertices such that any $k$-coloring of vertices of $G$ produces a rainbow induced copy of $H$. The results we obtain have a flavor quite different from of those answering Questions M and M-R. In particular, there are few exceptional graphs for which function $f$ is not finite.

Let $\Lambda$ be a graph on 4 vertices with exactly two adjacent edges and one isolated vertex. Let $K_{n}, E_{n}, S_{n}$ be a complete graph, an empty graph and a star on $n$ vertices, respectively. We define a class of graphs

$$
\mathcal{F}=\left\{K_{n}, E_{n}, S_{n}, \bar{S}_{n}, \Lambda, \bar{\Lambda}: n \in \mathbb{N}\right\}
$$

Note that any graph on at most three vertices is in $\mathcal{F}$. 
Theorem 4. Let $H$ be a graph on $k$ vertices. If $H \in \mathcal{F}$ then $f(H)=\infty$, otherwise $f(H) \leq 4 k-2$.

Corollary 1. Let $H$ be a graph on $k$ vertices, $H \notin \mathcal{F}$. For every graph $G$ on at least $4 k-1$ vertices there is a $k$-vertex coloring of $G$ avoiding rainbow induced subgraphs isomorphic to $H$.

\section{Proof of Theorem 4}

Let $H$ be a graph on $k$ vertices and let $\mathcal{I} n(H)$ be the set of graphs on at most $k-1$ vertices which are isomorphic to induced subgraphs of $H$.

One of our tools is the following theorem of Akiyama, Exoo and Harary, later strengthened by Bosák.

Proposition 1 ( $[\mathbf{1}],[4])$. Let $G$ be a graph on $n$ vertices such that all induced subgraphs of $G$ on $t$ vertices have the same size. If $2 \leq t \leq n-2$ then $G$ is either a complete graph or an empty graph.

Proposition 2. Let $H$ be a graph on $k$ vertices. If $G$ is a graph on at least $k$ vertices such that $G$ has an induced subgraph on at most $k-1$ vertices not isomorphic to any graph from $\mathcal{I} n(H)$, then there is a k-coloring of $G$ with no rainbow induced copy of $H$.

Proof. Let a set, $S$, of at most $k-1$ vertices in $G$ induce a graph not in $\mathcal{I} n(H)$. Color the vertices of $S$ with colors $1,2, \ldots,|S|$ and assign all colors from $\{|S|+1, \ldots, k\}$ to other vertices arbitrarily. Any rainbow subgraph of $G$ on $k$ vertices must use all of the vertices from $S$, but these vertices do not induce a subgraph of $H$. Therefore there is no rainbow induced copy of $H$ in this vertex-coloring of $G$.

We call a graph $G, H$-good if any induced subgraph of $G$ on at most $|V(H)|-1$ vertices is isomorphic to some graph from $\mathcal{I} n(H)$.

Corollary 2. Let $H \notin \mathcal{F}$ be a regular graph on $k$ vertices. Then $f(H)=k$.

Proof. Note that each graph in $\mathcal{I} n(H)$ on $k-1$ vertices has the same size. Let $G$ be a graph on $k+1$ vertices. By Proposition 2 we can assume that $G$ is $H$-good. Thus all $(k-1)$-subgraphs of $G$ have the same size. It follows from Proposition 1 that $G$ is either a complete or an empty graph. Therefore $G$ does not contain $H$ as an induced subgraph and any $k$-coloring of $G$ does not result in a rainbow induced copy of $H$.

We use the following notations for a graph $H=(V, E)$. Let $\alpha(H)$ be the size of the largest independent set of $H$, let $\omega(H)$ be the order of the largest complete subgraph of $H$. Let $\delta(H), \Delta(H)$ be the minimum and the maximum degrees of $H$ respectively. For two vertices $x, y$, such that $\{x, y\} \notin E, e=\{x, y\}$ is a non-edge, for a vertex $v, d(v)$ and $c d(v)$ are the degree and the codegree of $v$, i.e., the number of edges and non-edges 
incident to $v$, respectively. A $(k-1)$-subgraph of $H$ is an induced subgraph of $H$ on $k-1$ vertices. For all other definitions and notations we refer the reader to [9].

Next several lemmas provide some preliminary results for the proof of Theorem 4 . We consider the graph $H$ according to the following cases:

a) $\alpha(H)=k-1$ or $w(H)=k-1$,

b) $2 \leq \delta(H) \leq \Delta(H) \leq k-3$,

c) $\delta(H) \leq 1$ or $\Delta(H) \geq k-2$.

The cases $\mathbf{a}$ ) and $\mathbf{b}$ ) give us easy upper bounds on $f(H)$, the case $\mathbf{c}$ ) requires some more delicate analysis. The first lemma follows immediately from the definition of function $f$.

Lemma 1. $f(H)=f(\bar{H})$.

Lemma 2. Let $H$ be a graph on $k$ vertices such that $2 \leq \delta(H) \leq \Delta(H) \leq k-3$. Then $f(H) \leq 2 k-6$.

Proof. If a graph $G$ has a vertex of degree at least $k-2$ or of codegree at least $k-3$, then $G$ contains a subgraph on $k-1$ vertices not in $\mathcal{I} n(H)$ and by Proposition 2, there is a $k$-coloring of $G$ avoiding rainbow induced copies of $H$. Therefore, if any $k$-coloring of $G$ contains a rainbow induced copy of $H$ then for $v \in V(G)$ we have $|V(G)| \leq d(v)+$ $c d(v)+1 \leq(k-3)+(k-4)+1=2 k-6$.

Lemma 3. Let $H \notin \mathcal{F}$ be a graph on $k$ vertices, such that $\alpha(H)=k-1$ or such that $w(H)=k-1$. Then $f(H)=k$, for $k \geq 5$ and $f(H)=k+2$ for $k=4$.

Proof. Let $H$ be a graph on $k$ vertices with $\alpha(H)=k-1, H \notin \mathcal{F}$. Then $H$ is a disjoint union of a star with $k^{\prime}$ edges and $k-k^{\prime}-1$ isolated vertices, $1 \leq k^{\prime} \leq k-2$.

Assume first that $k \geq 5$. Let $G$ be a graph on $n$ vertices, $n \geq k+1$. If $G$ has two nonadjacent edges $e, e^{\prime}$, or a triangle, or no edges at all, by Proposition 2 there is a coloring of $G$ avoiding a rainbow induced copy of $H$. Therefore, $G$ must be a disjoint union of a star $S$ with $l$ edges and $n-l-1$ isolated vertices, $1 \leq l \leq n-1$. Then either $l>k^{\prime}$ or $n-l-1>k-k^{\prime}-1$. If $l>k^{\prime}$, we can use colors from $\left\{1, \ldots, k^{\prime}+1\right\}$ on the vertices of $S$ and colors from $\left\{k^{\prime}+2, \ldots, k\right\}$ on isolated vertices of $G$. If $n-l-1>k-k^{\prime}-1$ then we can use colors from $\left\{1, \ldots, k-k^{\prime}\right\}$ on isolated vertices of $G$ and other colors on the vertices of $S$. These colorings do not contain an induced rainbow subgraph isomorphic to $H$.

Let $k=4$. Since $H \notin \mathcal{F}$, we have that $H$ is a disjoint union of an edge and two vertices. If a graph $G$ has two adjacent edges $e, e^{\prime}$, we are done by Proposition 2. Otherwise, $G$ is a vertex disjoint union of isolated edges and vertices. Lets color $G$ so that the adjacent vertices get the same color. This coloring does not contain an induced rainbow copy of $H$. Moreover, if $|V(G)| \geq 7$ then there is such a coloring using 4 colors. Thus, $f(H)<7$. On the other hand, any 4-coloring of a graph $G$ consisting of three disjoint edges gives a rainbow induced $H$, thus $f(H) \geq 6$. We have then that $f(H)=6$.

If $w(H)=k-1$, Lemma 1 implies the same result. 
Lemma 4. Let $H$ be a graph on $k$ vertices, $H \notin \mathcal{F}, \alpha(H)<k-1, \omega(H)<k-1$. If $H$ has at least two nontrivial components then $f(H) \leq 2 k-1$.

Proof. Note that if $H$ has at least two nontrivial components and $\delta(H) \geq 2$, then we are done by Lemma 2. Let $m$ be the largest order of a connected component in $H$. Let $G$ be a graph on $n \geq 2 k$ vertices. We can assume by Proposition 2 that $G$ is $H$-good. Then there is no component in $G$ of order larger than $m$. Moreover, since $H$ is contained in $G$ as an induced subgraph, all components of $H$ of order $m$ appear in $G$ as connected components. Let $F_{1}, F_{2}, \ldots, F_{t}$ be components of $G$ of order $m$, let $x_{i}, y_{i} \in V\left(F_{i}\right), i=1, \ldots, t$. Assign color $i$ to both vertices $x_{i}$ and $y_{i}, i=1, \ldots, t$, and assign all colors from $\{t+1, \ldots, k\}$ to other vertices arbitrarily. Since $k \leq n / 2, t \leq n / 2$, we have that $t+k \leq n$ and such coloring exists. Consider a copy of $H$ in $G$. It contains at least one of the components of order $m$, thus it has at least two vertices of the same color. Therefore there is no rainbow induced subgraph of $G$ isomorphic to $H$ in this coloring.

Lemma 5. Let $H \notin \mathcal{F}$ be a graph on $k$ vertices such that $\delta(H) \leq 1, \alpha(H)<k-1$ and $w(H)<k-1$. Then $f(H) \leq 4 k-2$.

Proof. Let $H$ be a graph on $k$ vertices, $H \notin \mathcal{F}$ such that $\alpha(H)<k-1$ and $\omega(H)<k-1$. Let $G$ be a graph on $n \geq 4 k-1$ vertices. We can assume by Proposition 2 that $G$ is $H$-good.

Claim 0. If all graphs from $\mathcal{I} n(H)$ on $k-1$ vertices with a spanning star are isomorphic or do not exist, then $\Delta(G) \leq k-1$. If all graphs from $\mathcal{I} n(H)$ on $k-1$ vertices with an isolated vertex are isomorphic or do not exist, then $\Delta(\bar{G}) \leq k-1$.

To prove the Claim, assume that all graphs from $\mathcal{I} n(H)$ on $k-1$ vertices with a spanning star are isomorphic. Consider $S$, a neighborhood of a vertex $v$ of maximum degree in $G$. Then, all subsets of $S$ of size $k-2$ induce isomorphic graphs. Therefore, if $|S| \geq k$ we have, by Proposition 1 , that $S$ induces an empty or a complete graph on at least $k$ vertices, a contradiction. Thus, $|S|=\Delta(v) \leq k-1$. If there is no graph from $\mathcal{I} n(H)$ on $k-1$ vertices with a spanning star and $G$ has a vertex $v$ of degree at least $k-2$, then $v$ and $k-2$ of its neighbors induce a subgraph with a spanning star on $k-1$ vertices, a contradiction. The second statement can be proved in the same manner, concluding the proof of Claim 0.

Case 1. $\delta(H)=0$.

We can assume by Lemma 4 that $H$ has exactly one nontrivial component. Observe that either there is no $(k-1)$-vertex subgraph of $H$ with a spanning star, or all such subgraphs are isomorphic. Thus, by Claim $0, \Delta(G) \leq k-1$. Consider two adjacent vertices of $G, u$ and $v$. There is a set $T$ of vertices, $|T| \geq n-2-2(k-1)=n-2 k$, such that neither $u$ nor $v$ is adjacent to any vertex in $T$. Observe also, that since $G$ has no independent set of size $k-1$, the largest size of an independent set induced by vertices of $T$ is at most $k-2$. Let $T^{\prime} \subset T$ induce the largest independent set in $G[T]$. Then, for each 
$x \in T \backslash T^{\prime}$, there is $x^{\prime} \in T^{\prime}$ such that $x x^{\prime} \in E(G)$. Since $\left|T \backslash T^{\prime}\right| \geq n-2 k-k+2 \geq k$, it is clear that we can build a subgraph of $G[T]$ on $k-3$ vertices with no isolated vertices using some vertices from $T \backslash T^{\prime}$ and some of their neighbors from $T^{\prime}$ (provided that $k \geq 5$ ). Together with $u v$ it forms a subgraph on $(k-1)$ vertices with at least two nontrivial components and no isolated vertices. But each disconnected subgraph of $H$ on $k-1$ vertices has an isolated vertex, a contradiction.

Let $k=4$. Since $\delta(H)=0$ and $\alpha(H)<3, H$ must be a disjoint union of an isolated vertex and $K_{3}$. But then $H \in \mathcal{F}$, which is impossible.

Case 2. $\delta(H)=1$.

Lets call the vertices of degree 1 , leaves. We can assume that $H$ is connected by Lemma 4.

Case 2.1. All leaves in $H$ have a common neighbor, $v$.

Then all $(k-1)$-subgraphs of $H$ which have an isolated vertex are isomorphic to $H-v$, thus, by Claim 0 , we have that $\Delta(\bar{G}) \leq k-1$. Note that all $(k-1)$-subgraphs of $H$ having two adjacent vertices of degree $k-2$ are either isomorphic or do not exist. Consider $x, y$, two adjacent vertices of $G$. Since the codegree of each vertex is at most $k-1$ we have that there is a set $S$ of vertices, $|S| \geq n-2-2(k-1) \geq k-1$, such that each vertex of $S$ is adjacent to $x$ and to $y$. Thus, all $(k-3)$-subsets of $S$ induce isomorphic graphs, and $S$ must induce a complete or an empty graph on at least $k-1$ vertices by Proposition 1 , a contradiction.

Case 2.2. There are at least two leaves in $H$ which do not have a common neighbor.

It is easy to see that either $H$ does not have a vertex of degree $k-2$ or all subgraphs of $H$ on $k-1$ vertices with a spanning star are isomorphic. Then, by Claim $0, \Delta(G) \leq k-1$. Consider a set $S$ of vertices of $G$ inducing $H$ and let $S^{\prime} \subseteq S$ correspond to the set of leaves in $H$. Let $l$ be the largest number of leaves in $H$ having a common neighbor, let $x(l)$ be the number of distinct vertices in $H$ each adjacent to $l$ leaves.

If $l \leq 2$ or $(l=3$ and $x(l)=1)$ then all $(k-1)$-subgraphs of $H$ with at least three isolated vertices either do not exist or isomorphic. Consider three pairwise nonadjacent vertices $w, w^{\prime}, w^{\prime \prime}$ in $G$. Since $\Delta(G) \leq k-1$, there are at least $n-3-3(k-1) \geq k-1$ vertices of $G$ non-adjacent to either of $w, w^{\prime}, w^{\prime \prime}$. This is either impossible, or these vertices must induce an independent set or a clique, a contradiction.

Thus, we can assume that there are at least two distinct vertices in $H$ adjacent to at least three leaves each. Let $u, u^{\prime} \in S$ correspond to these vertices, and let $s, s^{\prime} \in N(u) \cap S$, $s^{\prime \prime} \in N\left(u^{\prime}\right) \cap S$. Since $V \backslash S$ has size at least $k-1$, it does not induce an independent set; thus there is an edge $v v^{\prime}, v, v^{\prime} \in V \backslash S$. If $v, v^{\prime}$ are not adjacent to any vertex in $S$, then $G\left[S \backslash\left\{s, s^{\prime}, s^{\prime \prime}\right\} \cup\left\{v, v^{\prime}\right\}\right]$ is a $(k-1)$-subgraph of $G$ with an isolated edge, no isolated vertices and with $\left|S^{\prime}\right|-1$ leaves. This is impossible, since each $(k-1)$-subgraph of $H$ with an isolated edge and no isolated vertices has at least $\left|S^{\prime}\right|$ leaves. If $v$ or $v^{\prime}$ is adjacent to some vertex $q \in S$ (we can always assume that $q \notin\left\{s, s^{\prime}, s^{\prime \prime}\right\}$ by choosing $s, s^{\prime}, s^{\prime \prime}$ accordingly), then $G\left[S \backslash\left\{s, s^{\prime}, s^{\prime \prime}\right\} \cup\left\{v, v^{\prime}\right\}\right]$ is a connected $(k-1)$-subgraph of $G$ 
with at most $\left|S^{\prime}\right|-2$ leaves. This is impossible since each connected subgraph of $H$ has at least $\left|S^{\prime}\right|-1$ leaves.

Now, we can quickly complete the proof of the main theorem using the result about the special graph $\Lambda$ proven in the next section.

Proof of Theorem 4. If $H=S_{k}$, then any $k$-coloring of $S_{n}, n \geq k$ induces a rainbow $H$. If $H=K_{k}$, then any $k$-coloring of $K_{n}, n \geq k$ induces a rainbow $H$. Using Proposition 3 for a graph $\Lambda$ and the fact that $f(H)=f(\bar{H})$ we have now established that for any $H \in \mathcal{F}$, $f(H)=\infty$.

Now, assume that $H$ is a graph on $k$ vertices, $H \notin \mathcal{F}$. If $\alpha(H)=k-1$ or $\omega(H)=k-1$, then, by Lemma 3, $f(H) \leq k+2$. If $\alpha(H)<k-1$ and $\omega(H)<k-1$ then at least one of the following holds:

1) $2 \leq \delta(H) \leq \Delta(H) \leq k-3$, and by Lemma $2, f(H) \leq 2 k-6$,

2) $\delta(H) \leq 1$, and by Lemmas 4 and $5, f(H) \leq 4 k-2$,

3) $\Delta(H) \geq k-2$, by 2) and Lemma $1, f(H) \leq 4 k-2$.

\section{Treating $\Lambda$}

Definition 5. Let $G(m)=(V, E)$,

$$
\begin{gathered}
V=\{v(i, j): \quad 1 \leq i \leq 7, \quad 1 \leq j \leq m\} \\
E=\{v(i, j) v(i+1, k): \quad 1 \leq j, k \leq m, j \neq k, 1 \leq i \leq 7\} \\
\{v(i, j) v(i+3, j): \quad 1 \leq j \leq m, 1 \leq i \leq 7\},
\end{gathered}
$$

addition is taken modulo 7 .

We have $V=V_{1} \cup \cdots \cup V_{7}=L_{1} \cup \cdots \cup L_{m}$, where $V_{i}=\{v(i, j): \quad 1 \leq j \leq m\}$, $1 \leq i \leq 7, L_{j}=\{v(i, j): \quad 1 \leq i \leq 7\}, 1 \leq j \leq m$. We shall refer to $V_{i}$ s as vertex parts and $L_{i}$ s as vertex layers. The edge-set of $G(m)$ can be constructed by first taking all the edges between consecutive (in cyclic order) $V_{i} \mathrm{~s}, i=1, \ldots, 7$ then removing the edges induced by each layer $L_{j}, j=1, \ldots, m$, and finally adding, for each $j=1, \ldots, m$, a new 7 cycle induced by $L_{j}$, see Figure 1 . Note that $G(1)$ is isomorphic to a 7-cycle, $G(2)$ has a spanning 14-cycle, and can be drawn as in the Figure 2.

Proposition 3. For any positive integer $m$ and any coloring of $V(G(m))$ into 4 colors, there is a rainbow induced subgraph of $G$ isomorphic to $\Lambda$.

Proof. We prove the statement, for $m=1,2,3$ and for $m>3$ use induction. This is a somewhat tedious but straightforward case analysis. 

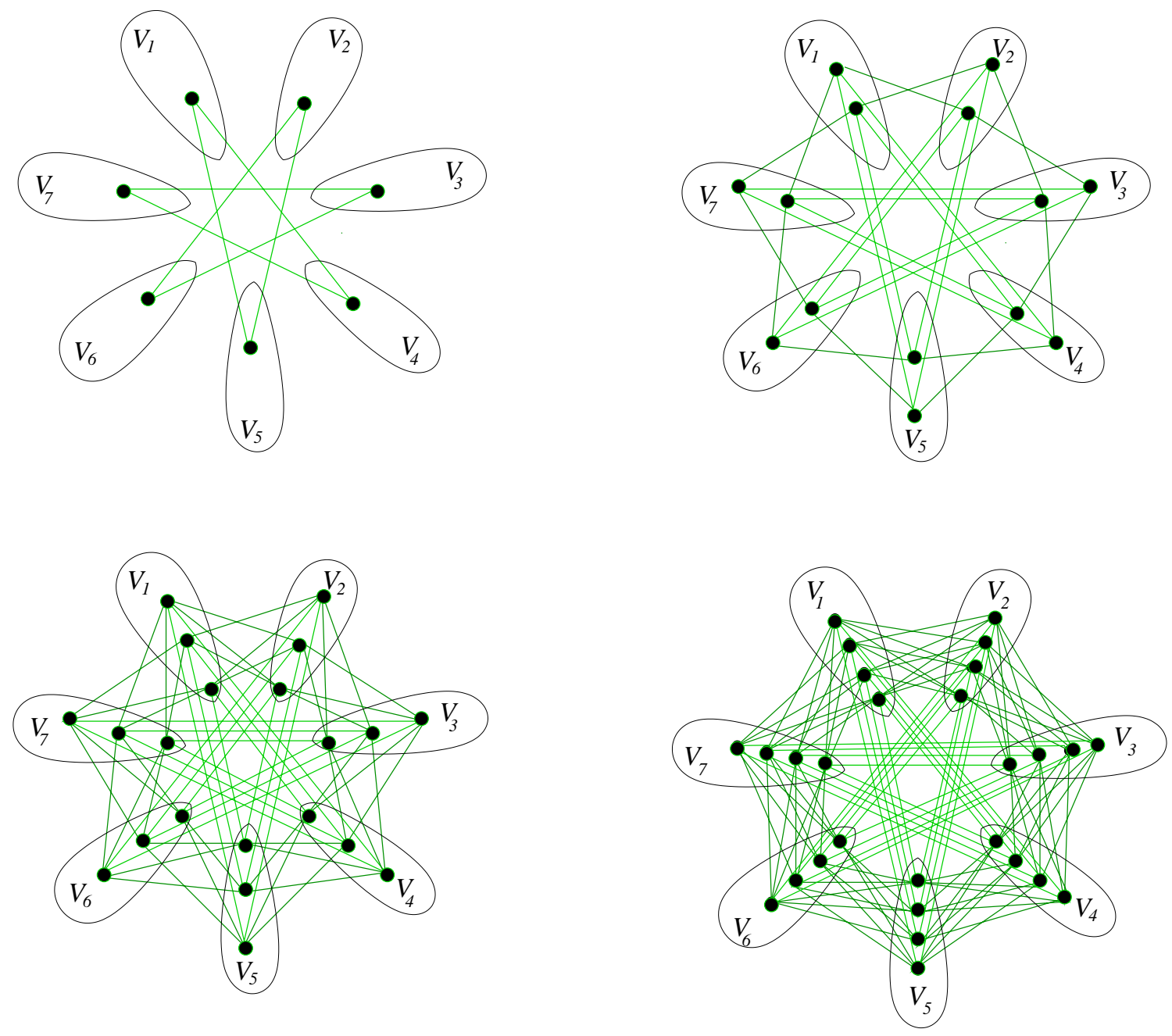

Figure 1: $G(1), G(2), G(3)$ and $G(4)$

Claim 1. Any coloring of $G(1)$ in 4 colors contains an induced rainbow $\Lambda$.

Let $G(1)$ have vertices $x_{1}, \ldots, x_{7}$ and edges $x_{i} x_{i+1}, i=1, \ldots, 7$, addition taken modulo 7. Assume that there is a 4-coloring $c$ with no induced rainbow $\Lambda$. First observe that any 4-coloring of $C_{7}$ must have three consecutive vertices with distinct colors, say $c\left(x_{i}\right)=i$, for $i=1,2,3$. Then $c\left(x_{5}\right) \neq 4, c\left(x_{6}\right) \neq 4$, thus, without loss of generality $c\left(x_{4}\right)=4$. Note that then $c\left(x_{7}\right) \neq 1, c\left(x_{7}\right) \neq 3$. If $c\left(x_{7}\right)=4$ then $x_{6}$ must have color 3 , and there is no color available for $x_{5}$. If $c\left(x_{7}\right)=2$ then $c\left(x_{6}\right)=2$ and there is no available color for $x_{5}$.

Claim 2. Any coloring of $G(2)$ in 4 colors contains an induced rainbow $\Lambda$.

Note that $G(2)$ can be drawn as $C_{14}$ with chords as in Figure 2. Let the vertices of $G(2)$ be $x_{1}, \ldots, x_{14}$ in order on the cycle and let the edges be $x_{i}, x_{i+1}, x_{i+4}, i=1, \ldots, 14$, where addition is taken modulo 14 . We shall use the fact that the following sets of vertices 


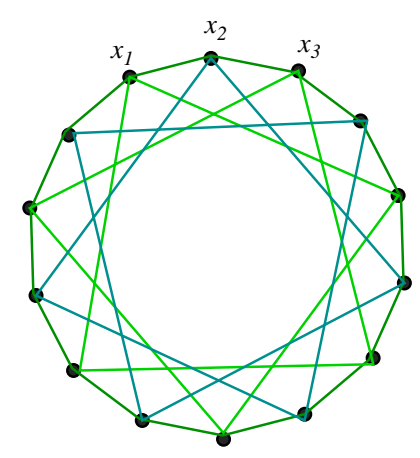

Figure 2: Different drawing of $G(2)$

induce $C_{7}$ and thus cannot use all 4 colors:

$$
\left\{x_{i}, \quad x_{i+2}, x_{i+3}, x_{i+4}, \quad x_{i-2}, x_{i-3}, x_{i-4}\right\},
$$

$i=1, \ldots, 14$ and addition is taken modulo 14 . We shall also use an easy fact that it is impossible to have a 4 -colored $C_{4}$ in $G(2)$.

Case 1. There are three consecutive vertices, using distinct colors, say $c\left(x_{i}\right)=i, i=1,2,3$.

Then, considering all induced cycles of length 7 containing these three vertices, we see that the only vertices which could have color 4 are $x_{4}, x_{6}, x_{14}$ or $x_{12}$.

Case 1.1. $c\left(x_{4}\right)=4$.

Consider vertex $x_{8}$. If $c\left(x_{8}\right)=1$ then $\left\{x_{2}, x_{3}, x_{4}, x_{6}, x_{8}, x_{9}, x_{10}\right\}$ induces a $C_{7}$ using 4 colors. If $c\left(x_{8}\right)=2$ then $\left\{x_{1}, x_{3}, x_{4}, x_{8}\right\}$ induces a rainbow $\Lambda$. If $c\left(x_{8}\right)=3$ then $\left\{x_{14}, x_{1}, x_{2}, x_{4}, x_{6}, x_{7}, x_{8}\right\}$ induces a $C_{7}$ using 4 colors. Thus $x_{8}$ cannot be assigned any color and this case is impossible.

Case 1.2. $c\left(x_{6}\right)=4$.

Consider vertex $x_{7}$. If $c\left(x_{7}\right)=1$ then $\left\{x_{2}, x_{3}, x_{6}, x_{7}\right\}$ is a 4-colored $C_{4}$. If $c\left(x_{7}\right)=2$ then $\left\{x_{1}, x_{3}, x_{7}, x_{6}\right\}$ induces a rainbow $\Lambda$. If $c\left(x_{7}\right)=3$ then $\left\{x_{14}, x_{1}, x_{2}, x_{4}, x_{6}, x_{7}, x_{8}\right\}$ induces a $C_{7}$ using 4 colors. Therefore $x_{7}$ cannot be assigned a color and this case is impossible as well.

By symmetry $c\left(x_{14}\right) \neq 4$ and $c\left(x_{12}\right) \neq 4$, so there is no vertex colored 4 , a contradiction.

Case 2. There are no three consecutive vertices using distinct colors.

Then, without loss of generality, there are consecutive vertices $x_{i}, x_{i+1}, \ldots, x_{j}$ such that $c\left(x_{i}\right)=a, c\left(x_{j}\right)=b$ and $c\left(x_{m}\right)=c$, for $i<m<j$, such that $a, b, c$ are distinct. Consider smallest such set of vertices and assume that $i=1, a=2, b=3, c=1$. Then clearly, $j \geq 4$, moreover $j \leq 5$ since otherwise there is a smaller such set.

Case 2.1. $j=4$.

By considering all induced $C_{7}$ containing vertices of colors $1,2,3$ from $\left\{x_{1}, x_{2}, x_{3}, x_{4}\right\}$, and using the fact that $x_{14}$ and $x_{5}$ cannot have color 4 without creating three consecutive 
vertices of distinct colors, we see that the only vertices which could have color 4 are $x_{9}$ and $x_{10}$. If $c\left(x_{10}\right)=4$ then consider vertex $x_{14}$. If $c\left(x_{14}\right)=3$ or 4 then $x_{14}, x_{1}, x_{2}$ are three consecutive vertices using distinct colors. If $c\left(x_{14}\right)=2$ then $\left\{x_{14}, x_{10}, x_{4}, x_{2}\right\}$ induces a rainbow $\Lambda$. Thus $c\left(x_{14}\right)=1$. Consider $x_{5}: c\left(x_{5}\right) \neq 4$ and $c\left(x_{5}\right) \neq 2$ since otherwise there are three consecutive vertices of distinct colors. If $c\left(x_{5}\right)=3$ then $\left\{x_{2}, x_{1}, x_{5}, x_{9}\right\}$ induces a rainbow $\Lambda$. If $c\left(x_{5}\right)=1$ then $\left\{x_{4}, x_{5}, x_{1}, x_{9}\right\}$ induces a rainbow $\Lambda$. Thus this case is impossible. If $c\left(x_{9}\right)=4$ we arrive at a contradiction by symmetry.

Case 2.2. $j=5$.

By considering all induced $C_{7}$ containing vertices of colors $1,2,3$ from $\left\{x_{1}, \ldots, x_{5}\right\}$ we see that the only vertex which might, and thus must have color 4 is $x_{10}$. But then $\left\{x_{10}, x_{1}, x_{2}, x_{5}\right\}$ induces a rainbow $\Lambda$, a contradiction.

Claim 3. Any coloring of $G(3)$ in 4 colors contains an induced rainbow $\Lambda$.

Let $c$ be a coloring of $G(3)$ using colors 1,2,3,4 and containing no induced rainbow copy of $\Lambda$. If there is a subgraph of $G(3)$ isomorphic to $G(2)$ and using four colors, there is a rainbow induced $\Lambda$ by Claim 2. Therefore, we can assume that each vertex layer of $G(3)$ has a color used only on its vertices and on no vertex of any other layer. In particular, assume that color $i$ is used only in $L_{i}, i=1,2,3$. So, $L_{1}$ uses colors from $\{1,4\}, L_{2}$ uses colors from $\{2,4\}$, and $L_{3}$ uses colors from $\{3,4\}$.

If there is a part, say $V_{1}$, using colors $1,2,3$, then it is easy to see that none of the vertices of $V_{2}$ could have color 4 and moreover $V_{2}$ must use all three colors 1,2,3 again, in respective layers. This shows that in this case all sets $V_{i}, i=1, \ldots, 7$ must use only colors $1,2,3$ and there is no vertex of color 4 , a contradiction. Since there is no part $V_{i}$, $i=1, \ldots, 7$ using all colors $1,2,3$, each part must have color 4 on some vertex.

Assume that there is a part, say $V_{1}$, having exactly one vertex of color 4 . Without loss of generality, we have $c(v(1,1))=4, c(v(1,2))=2, c(v(1,3))=3$, then $c(v(7,1))=$ $c(v(2,1))=4$. Moreover, $c(v(i, 1)) \neq 1$ for $i=3,4,5,6$, otherwise one of these vertices together with either $\{v(2,1), v(1,2), v(1,3)\}$ or with $\{v(7,1), v(1,2), v(1,3)\}$ induces a rainbow $\Lambda$. Therefore, there is no vertex of color 1 in the graph, a contradiction.

Thus, each part $V_{i}$ has at least two vertices of color 4 . Then, it is easy to see that there is always a rainbow induced $\Lambda$ in such a coloring of $G(3)$, a contradiction.

Induction step. Assume that $m \geq 4$. If there is a vertex layer $L_{i}$ such that $G\left[V-L_{i}\right]$ uses all 4 colors, then, since $G\left[V-L_{i}\right]$ is isomorphic to $G(m-1)$, there is a rainbow induced subgraph isomorphic to $\Lambda$. Thus we can assume that each layer $L_{1}, L_{2}, \ldots, L_{m}$ uses a color not present in other layers. It is possible only if $m=4$, in which case all vertices of each layer have the same color. We can assume that all vertices of layer $L_{i}$ have color $i, i=1,2,3,4$. But then it is easy to see that there is an induced rainbow $\Lambda$ in this coloring. 
It is interesting to see that if $G$ is a bipartite graph then there is always a coloring of $V(G)$ in 4 colors avoiding induced rainbow $\Lambda$. Indeed, if $G$ is a complete bipartite graph, it does not have any induced copies of $\Lambda$, so any 4-coloring will work. Thus, we can assume that there are two nonadjacent vertices from different partite sets $A$ and $B, x \in A$ and $y \in B$. Let $c(x)=3, c(y)=4, c(N(x))=1, c(N(y))=2, c(A \backslash(N(y) \cup\{x\}))=1$ and $c(B \backslash(N(x) \cup\{y\}))=2$. It is easy to see that this coloring does not have a rainbow induced $\Lambda$.

Concluding Remark: We have proven that for any graph $H \notin \mathcal{F}$ on $k$ vertices and any graph $G$ on $4 k-1$ vertices there is a coloring of $G$ in $k$ colors avoiding rainbow induced subgraph isomorphic to $H$. Together with definition of $f$, this implies that

$$
k \leq \max \{f(H):|V(H)|=k, H \notin \mathcal{F}\} \leq 4 k-2 .
$$

There are many classes of graphs for which $f(H)=k$, which follows, for example, from Proposition 2. We believe that the above upper bound could be improved to $2 k-1$ with a more careful analysis, and, perhaps to $k+c$, where $c$ is a constant. As far as the lower bound is concerned, we have only one example when $f(H)=k+2$ for $k=4$, provided by Lemma 3. It will be very interesting to see constructions of graphs giving better lower bounds on $f$.

\section{References}

[1] Akiyama, J., Exoo, G., Harary, F., The graphs with all induced subgraphs isomorphic, Bull. Malaysian Math. Soc. (2), 2 (1979), no. 1, 43-44.

[2] Borowiecka-Olszewska, M., Drgas-Burchardt, E., Mihók, P., Minimal vertex Ramsey graphs and minimal forbidden subgraphs, Discrete Math., 286 (2004), no. 1-2, 31-36.

[3] Brown, J., Rödl, V., A Ramsey type problem concerning vertex colorings, J. Combin. Theory Ser. B, 52 (1991), no. 1, 45-52.

[4] Bosák, J., Induced subgraphs, Finite and infinite sets, Vol. I, II (Eger, 1981), Colloq. Math. Soc. János Bolyai, 37, North-Holland, Amsterdam (1984), 109-118.

[5] Deuber, W. A., Canonization, Combinatorics, Paul Erdős is eighty, Bolyai Soc. Math. Stud., János Bolyai Math. Soc., Budapest, 1 (1993), 107-123,

[6] Eaton, N., Rödl, V., A canonical Ramsey theorem, Random Structures Algorithms, 3 (1992), no. 4, 427-444.

[7] Graham, R., Rothschild, B., Spencer, J., Ramsey theory, Second edition. WileyInterscience Series in Discrete Mathematics and Optimization, New York, 1990.

[8] Łuczak, T., Rucinski, A., Urbanski, S., Vertex Ramsey properties of families of graphs, J. Combin. Theory Ser. B, 84 (2002), no. 2, 240-248.

[9] West, D., Introduction to Graph Theory, Second Edition, Prentice Hall, 2001. 